https://doi.org/10.15407/ujpe64.12.1108

YU.A. KUROCHKIN

B.I. Stepanov Institute of Physics of the National Academy of Sciences of Belarus

(68/2, Nezavisimosti Ave., Minsk 220072, Belarus; e-mail: yukuroch@dragon.bas-net.by)

\title{
FREE MOTION OF PARTICLES \\ IN THE LOBACHEVSKII SPACE IN TERMS OF THE SCATTERING THEORY ${ }^{1}$
}

\begin{abstract}
The problem of the motion of a free particle in the three-dimensional Lobachevskii space are interpreted as scattering by the space. The quantum-mechanical case is considered on the basis of the integral equation derived from the Schrödinger equation. After the separation of variables in a quasi-Cartesian coordinate system, the integral equation is derived for the momentum component along the axis of symmetry of a horosphere, which coincides with the $z$ axis. The relationship between the scattering amplitude and analytical functions is established. The methods of iteration and finite differences are used to solve the integral equation.

Keywords: Lobachevskii space, horosphere, Fourier transform, coordinates, Schrödinger equation, scattering, amplitude of scattering, analytical representation, method, iterations, finite differences.
\end{abstract}

\section{Introduction}

In [1], the motion of a classical or quantum-mechanical free particle in the three-dimensional Lobachevskii space was interpreted as the scattering by the space itself. At the same time, the solution of the quantum-mechanical problem was based on the exact solution of the Schrödinger equation in the configuration space in quasi-Cartesian coordinates, which are essentially associated with the horospheres [2]. Below, the same quantum-mechanical problem will be solved using the usual Fourier transformation of the original equation.

Note that the Fourier transformation in the Lobachevskii space is understood most often as the Shapiro transformation [3] or the Gelfand-Graev transformation [4], which is actually the same (see also [5]). We will use the usual Fourier transformation, which ultimately leads to the formulation of a one-dimensional integral equation for the momentum component along the axis of symmetry of the horosphere after the separation of variables.

In view of the considerable convenience of the use of integral equations in scattering theory such as, for example, the Lippmann-Schwinger equation (see, e.g., $[6]$ ), the Logunov-Tavkhelidze-Kadyshevsky equation [7], and others, we will specially pay attention

(C) YU.A. KUROCHKIN, 2019

\section{8}

to the analysis of a particle motion in the Lobachevskii space in terms of the integral equation obtained below.

We also note that the problem of the propagation of electromagnetic waves and particles was considered in [8-10], where the effect of a "mirror" was established, i.e., the reflection of waves and particles under certain propagation conditions. The effect is explained by the fact that, in the direction of the propagation of waves (particles), the space in the horospherical or quasi-Cartesian coordinates imitates a nonuniform medium that inhibits their propagation and ultimately leads to the stopping and the reflection of waves.

\section{Quantum-Mechanical Problem. Derivation of an Integral Equation}

Let us consider the motion of a free quantum-mechanical particle in the three-dimensional Lobachevskii space as the scattering. To do this, starting from the Schrödinger equation in the quasi-Cartesian coordinates, we formulate the problem for the integral equation. We write the Schrödinger equation

$i \hbar \frac{\partial \Psi}{\partial t}=H \Psi$

1 This work is based on the results presented at the XI BolyaiGauss-Lobachevskii (BGL-2019) Conference: Non-Euclidean, Noncommutative Geometry and Quantum Physics.

ISSN 2071-0194. Ukr. J. Phys. 2019. Vol. 64, No. 12 
in the quasi-Cartesian coordinates of the threedimensional Lobachevskii space with the metric

$d l^{2}=\rho^{2}\left[e^{2 z}\left(d x^{2}+d y^{2}\right)+d z^{2}\right]$

and with Hamiltonian

$H=-\frac{\hbar^{2}}{2 m \rho^{2}}\left[e^{2 z}\left(\frac{\partial^{2}}{\partial x^{2}}+\frac{\partial^{2}}{\partial y^{2}}\right)+e^{2 z} \frac{\partial}{\partial z} e^{2 z} \frac{\partial}{\partial z}\right]$.

The stationary problem

$H \Psi=E \Psi$

for Eq. (1) with regard for Hamiltonian (3) can be formulated as follows:

$\left[\left(\frac{\partial^{2}}{\partial x^{2}}+\frac{\partial^{2}}{\partial y^{2}}\right)+\frac{\partial}{\partial z} e^{2 z} \frac{\partial}{\partial z}\right] \Psi(x, y, z)=$

$=e^{-2 z} k^{2} \Psi(x, y, z)$,

where

$k^{2}=-\frac{2 m E \rho^{2}}{\hbar^{2}}$.

In formula (6), $m$ is the mass of the particle, $E$ is its energy, and $\rho$ is the radius of curvature of the space.

For problem (4), which is reduced to the solution of Eq. (5), it is possible to separate the variables:

$\left(\frac{\partial^{2}}{\partial x^{2}}+\frac{\partial^{2}}{\partial y^{2}}\right) \chi(x, y)=k_{\perp}^{2} \chi(x, y)$,

$\frac{\partial}{\partial z} e^{2 z} \frac{\partial}{\partial z} w(z)=\left(k^{2} e^{-2 z}-k_{\perp}^{2}\right) w(z)$.

Here, we presented the wave function as $\Psi(x, y, z)=$ $\chi(x, y) w(z)$.

For our purposes, it is convenient to present Eq. (8) in the form

$\frac{d^{2} w}{d z^{2}}-2 \frac{d w}{d z}-\left(k^{2}-1\right) w=-k_{\perp}^{2} e^{2 z} w$

or, as a result of the standard transformation and the exclusion of the first derivative,

$\left(\frac{d^{2}}{d z^{2}}-k^{2}\right) \varphi=-k_{\perp}^{2} e^{2 z} \varphi$.

As a solution to Eq. (7), we choose a plane wave defined by the components $p_{x}$ and $p_{y}$ of the wave vector, where $p_{x}^{2}+p_{y}^{2}=k_{\perp}^{2}$.

ISSN 2071-0194. Ukr. J. Phys. 2019. Vol. 64, No. 12
Now, we present the wave function in Eq. (9) in the form of the Fourier transform

$\varphi(z)=\int_{-\infty}^{\infty} \frac{\widetilde{\varphi}\left(p_{z}\right)}{\sqrt{\pi}} e^{-i z p_{z}} d p_{z}$

As a result, we get the integral equation

$\widetilde{\varphi}\left(p_{z}\right)=\frac{k_{\perp}^{2}}{-p_{z}^{2}-k^{2}} \int_{-\infty}^{\infty} \frac{\widetilde{\varphi}\left(p_{z}^{\prime}\right)}{p_{z}^{\prime}-p_{z}-2 i} d p_{z}^{\prime}$

Equation (12) allows us to analyze the conditions for the occurrence of bound states in the extended Lobachevskii space, as well as to describe the scattering under the action of the effective potential arising in the Lobachevskii space, understood as a change in $p_{z}$.

The dependence $p_{z}(z)=\sqrt{k^{2}-k_{\perp}{ }^{2} e^{2 z}}$ follows, in particular, from the solution of the Hamilton-Jacobi equation given in [1].

For the latter case, Eq. (12) takes the form

$\widetilde{\varphi}\left(p_{z}\right)=\delta\left(p_{z}-\kappa\right)+\frac{k_{\perp}^{2}}{-p_{z}^{2}-k^{2}} \int_{-\infty}^{\infty} \frac{\widetilde{\varphi}\left(p_{z}^{\prime}\right)}{p_{z}^{\prime}-p_{z}-2 i} d p_{z}^{\prime}$.

\section{Analysis of the Integral Equation and Its Solutions}

As is known, the bound states are determined by the poles of the wave function in the complex plane and correspond to negative energy values. In this case, the preintegral expression in the second term of formula (12) defines the Green function of Eq. (9); it shows a pole for the energy value

$E=\frac{\hbar^{2} p_{z}^{2}}{2 m \rho^{2}}$

From expression (14), it can be seen that it can take negative values only in the space of positive curvature, which is realizable only in the imaginary Lobachevskii space (in the three-dimensional de Sitter space). The developed approach is applicable to the description of the motion of a quantum-mechanical particle in a given space under similar initial conditions. It is enough to make a replacement $\rho^{2} \rightarrow-\rho^{2}$ in (14). The use of other methods confirms the existence of bound states of a free particle (the discrete 
part of the spectrum of states) in the imaginary Lobachevskii space, despite the non-compactness of this space. The scattering problem with Eq. (13) will be solved by the method of iterations. To do this, we substitute the original plane wave $\delta\left(p_{z}-\kappa\right)$ corresponding to the zero approximation to the second term and obtain the first (Born) approximation

$\widetilde{\varphi}\left(p_{z}\right)=\delta\left(p_{z}-\kappa\right)-\frac{k_{\perp}^{2}}{p_{z}^{2}+k^{2}} \frac{1}{\kappa-p_{z}-2 i}$.

Note that the modulus of the wave vector $\kappa$ of a plane wave which came from infinity satisfies Eq. (10) without the right-hand side. We accept that this wave vector is directed along the $z$-axis of a beam of the classical problem.

The matrix element of the scattering amplitude in expression (15) is equal to

$M_{\kappa \rightarrow p_{z}}=\frac{k_{\perp}^{2}}{\kappa-p_{z}-2 i}$.

The subsequent iterations give a zero contribution.

\section{Analytic Representation of the Solution}

As is known [11], for a function satisfying the condition of quadratic integrability on the entire number axis

$\int_{-\infty}^{\infty}\left|\varphi\left(p_{z}\right)\right|^{2} d p_{z}<\infty$

there is an analytic representation

$f(z)=\frac{1}{2 i \pi} \int_{-\infty}^{\infty} \frac{\widetilde{\varphi}\left(p_{z}^{\prime}\right) d p_{z}^{\prime}}{p_{z}^{\prime}-z}$

Then the integral in expression (12) is obviously a special case of integral (18), and the corresponding analytic function has a fixed value of the imaginary part of the argument in this case. In this case, Eq. (10) takes the form

$\varphi\left(p_{z}\right)=\frac{2 i \pi k_{\perp}^{2}}{-p_{z}^{2}-k^{2}} f\left(p_{z}+2 i\right)$.

Expression (19) is conveniently solved within the method of finite differences. Indeed, let us set $p_{z}=0$. Then

$\widetilde{\varphi}(0)=-\frac{2 i \pi k_{\perp}^{2}}{k^{2}} f(2 i)$.

1110
We take $\varphi(0)$ as the given boundary value and shift the real part of the argument by a small finite value $\delta, \delta \ll 1$. On the next step, we have

$\widetilde{\varphi}(\delta)=\frac{2 i \pi k_{\perp}^{2}}{-\delta^{2}-k^{2}} f(\delta+2 i)$.

Expanding $f(\delta+2 i)$ in a Taylor series with respect $\delta$ and taking the Cauchy-Riemann relations into account, we obtain $f(\delta+2 i)=f(2 i)$ and, as a result, the expression

$\widetilde{\varphi}(\delta)=\frac{2 i \pi k_{\perp}^{2}}{-\delta^{2}-k^{2}} f(2 i)$.

Continuing the iteration, it is easy to verify that

$\widetilde{\varphi}(n \delta)=\frac{2 i \pi k_{\perp}^{2}}{-n^{2} \delta^{2}-k^{2}} f(2 i)$.

Here, $n$ is an integer and the iteration order. Replacing $f(2 i)$ in (23) according to formula (20), we finally obtain

$\widetilde{\varphi}(n \delta)=\frac{k^{2}}{n^{2} \delta^{2}+k^{2}} \widetilde{\varphi}(0)$.

Expression (24) is consistent, because it becomes the identity, when $n=0$. The results for a relativistic scalar particle follow from those obtained above by replacing

$2 m E \rightarrow \frac{E^{2}}{c^{2}}-m^{2} c^{2}$

in formula (6). Here, $c$ is the speed of light.

\section{Conclusion}

Thus, the problem of the motion of a free particle in the three-dimensional Lobachevskii space is interpreted as a scattering problem. The quantum-mechanical case is considered on the basis of the integral equation. The results can be used to construct phenomenological models of hadrons and to solve problems of astrophysics and cosmology.

1. Yu.A. Kurochkin. Interpretation of the free motion of particles in the Lobachevskii space in terms of the scattering theory. Vesci NAN Belar. Ser. Fiz.-Mat. Nav. No. 3, 49 (2017).

2. M.N. Olevskii. Three orthogonal systems in the spaces of constant curvature in which equation allows full separation of variables. Matem. Sborn. 27, 379 (1950).

ISSN 2071-0194. Ukr. J. Phys. 2019. Vol. 64, No. 12 
3. I.S. Shapiro. Decomposition of the wave function on irreducible representations of the Lorentz group. DAN SSSR 106, 647 (1956).

4. I.M. Gelfand, M.I. Graev, N.Ya. Vilenkin. Generalized Functions. 5. Integral Geometry and Representation Theory (Academic Press, 1966).

5. N.Ya. Vilenkin, Ya.A. Smorodinskii. Invariant decomposition of the relativistic amplitude. Zh. Eksp. Teor. Fiz. 46, 1793 (1964).

6. V.G. Kadyshevskii, R.M. Mir Kasimov, N.B. Skachkov. Three-dimensional formulation of the relativistic two-body problem. Part. Nucl. 2, 635 (1972).

7. Ta-You Wu, Takashi Ohmura. Quantum Theory of Scattering (Prentice-Hall, 1962).

8. E.M. Ovsiyuk. Exactly Solved Problems of Quantum Mechanics and Classical Theory of the Field in Space with Non-Euclidean Geometry (RIVSh, 2013).

9. E.M. Ovsiyuk, O.V. Veko, V.M. Red'kov. On simulating a medium with special reflecting properties by Lobachevskii geometry. Nonlin. Phen. Complex Syst. 16 (4), 331 (2013).

10. E.M. Ovsiyuk, O.V. Veco. About modeling of the potential barrier in the Schr'odinger theory by Lobachevskii space geometry. Vestn. Brest. Univ. Ser. 4. Fiz., Mat. No. 2, 30 (2011).

11. H.J. Bremermann. Distributions, Complex Variables, and Fourier Transforms (Addison-Wesley, 1965).

Received 02.09.19

Ю.А. Курочкін

ВІЛЬНИЙ РУХ ЧАСТИНОК

У ПРОСТОРІ ЛОБАЧЕВСЬКОГО

B TEPMIHAX TEOPIÏ РОЗСІЯННЯ

$\mathrm{P}$ е $з$ ю м е

Рух вільних частинок у тривимірному просторі Лобачевського трактується як розсіяння у просторі. Квантовомеханічний випадок розглядається на основі інтегрального рівняння, отриманого з рівняння Шредінгера. Після розділення змінних у квазідекартовій системі координат виводиться інтегральне рівняння для компоненти імпульсу вздовж осі симетрії горосфери, яка збігається з віссю $z$. Встановлено взаємозв'язок амплітуди розсіяння та аналітичних функцій. Використано методи ітерацій та кінцевих приростів для розв'язання інтегрального рівняння. 